\title{
Numerical simulation of bubble dynamics and heat transfer with transient thermal response of solid wall during pool boiling of FC-72
}

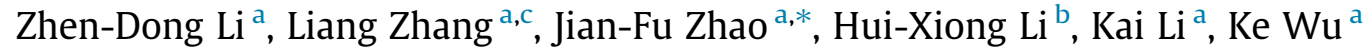 \\ ${ }^{a}$ Key Laboratory of Microgravity (National Microgravity Laboratory)/CAS, Institute of Mechanics, Chinese Academy of Sciences (CAS), Beijing 100190, China \\ ' State Key Laboratory of Multiphase Flow in Power Engineering, Xi'an Jiaotong University, Xi'an 710049, China \\ 'State Nuclear Power Software Development Center, Beijing 102209, China
}

\section{A R T I C L E I N F O}

\section{Article history:}

Received 22 September 2014

Received in revised form 23 December 2014

Accepted 23 December 2014

Available online 22 January 2015

\section{Keywords:}

Transient thermal response

Single bubble pool boiling

Multi-cycle simulation

Quasi-steady boiling

\begin{abstract}
A B S T R A C T
Using the Ghost Fluid Method for sharp interface representation, bubble dynamics and heat transfer during single bubble pool boiling of saturated FC-72 are simulated numerically with transient thermal response of the heated $\mathrm{SiO}_{2}$ solid wall. A constant and uniform temperature is fixed on to the bottom surface of the solid wall in the simulations, and thus, both the spatio-temporal averaged heat flux and superheat on the top surface, which contacts the working fluid directly, are dependent variables instead of controllable ones. Multi-cycle simulations are carried out to eliminate the influence of unreal initial conditions. Steady periodical processes of single bubble pool boiling can be reached on the wall with a thickness of $1 \mathrm{~mm}$, while only quasi-steady ones on the wall with a thickness of $5 \mathrm{~mm}$ due to the limited simulation time, which results in a smaller thermal penetration depth inside the solid wall compared with its thickness. Comparing with the prediction of the correlation by Zuber for the discrete bubble region and experimental data of single bubble pool boiling, the numerical results of boiling curves in both steady and quasi-steady cases exhibit the same trend. Transient heat conduction inside the solid wall is analyzed in detail. A sharp drop of the wall temperature is evident in the vicinity of the contact line due to violent evaporation in this tiny region. The area of the temperature drop moves with the contact line, resulting in a pseudo-periodical process of heat storage and release inside the solid wall, which exhibits a coupling effect with bubble dynamics and heat transfer. The thermal penetration depth caused by the processes of bubble growth and departure in a single bubble cycle is about $0.5 \mathrm{~mm}$ in both steady and quasi-steady cases, which is much small than the heater thickness.
\end{abstract}

(c) 2014 Elsevier Ltd. All rights reserved.

\section{Introduction}

Nucleate boiling is widely used in industry because it allows transferring high heat fluxes at moderate wall superheats with the release of latent heat. In order to make better use of this highly efficient heat transfer process, the mechanisms of the phenomenon have been intensively studied in the last decades, and are still subject of many ongoing research activities all over the world. With many scientists' contributions, great strides have been made towards a fundamental understanding of the process.

According to the present results, the heat transfer mechanisms of nucleate boiling can be subdivided into two categories, namely convective heat transfer and latent heat transfer. The convective heat transfer takes effect in the bulk liquid region, and includes

\footnotetext{
* Corresponding author.

E-mail address: jfzhao@imech.ac.cn (J.-F. Zhao).
}

several much fine mechanisms, such as natural convective heat transfer resulted from fluid thermal inhomogeneity, enhanced convective heat transfer induced by bubble rising motion $[1,2]$, and transient heat conduction within the superheated liquid layer restoring process by conductive heat transfer from the heater into the liquid during bubble detachment and waiting period [3]. The latent heat transfer takes effect in the liquid-vapor interface region, which can be divided into the following two parts. One is the evaporation of bulk superheated liquid around the growing bubble [4], while the other is related with phase change along the surface of liquid between the growing bubble and the superheated wall. For the latter case, there isn't a common accepted model up to now. However, two models, i.e. the microlayer evaporation model [5] and the 3-phase contact line evaporation model [6], were often used in the literature. The major difference between them is the size of the liquid film (thicker than molecular level) on the heat transfer surface. In the first one, the liquid film, or microlayer, exists in the entire region underneath the bubble, 


\section{Nomenclature}

A area

$C_{p} \quad$ specific heat

D diameter

g gravity vector

$H \quad$ Heaviside function

$h \quad$ grid size

$h_{e v} \quad$ interfacial thermal resistance

$h_{f g} \quad$ latent heat

$k$ thermal conductivity

L characteristic or Laplace length

$\dot{m} \quad$ mass flux

n normal vector

$p \quad$ pressure

$q \quad$ heat flux

$R_{1} \quad$ end position of micro region

$R_{0} \quad$ three phase contact point

$T$ temperature

$t$ time

t tangential vector

$U \quad$ characteristic velocity

u velocity vector

$u \quad r$-directional velocity

V volume

$v \quad z$-directional velocity

$\Delta T \quad$ superheat

Greece symbols

$\alpha \quad$ thermal diffusion

$\beta_{T} \quad$ interfacial thermal expansion $\gamma \quad$ apparent contact angle

$\delta \quad$ thickness of micro region

$\delta_{T} \quad$ thermal boundary layer thickness

$\theta \quad$ characteristic temperature

$\kappa \quad$ curvature

$\Lambda \quad$ relevant physical parameters

$\mu \quad$ dynamic viscosity

$v \quad$ kinematic viscosity

$\rho \quad$ density

$\sigma \quad$ surface tension

$\varphi \quad$ level set function for vapor-liquid interface

$\psi \quad$ level set function for liquid-solid interface

$\Omega \quad$ phase domain

$\Gamma \quad$ interface between phases

Subscripts

c bubble cycle

$d$ departure

$f \quad$ fluid

$h \quad$ heater

int interface

$l \quad$ liquid

mic micro-region

$n \quad$ nucleation

$s \quad$ solid wall

sat saturation

$v \quad$ vapor

$w \quad$ wall or waiting time

ave spatio-temporal averaged while in the latter just in a tiny space, or micro region, adjacent to the bubble base. Corresponding to the two models, the evaporation occurs in the entire region underneath the bubble or just occurs near the bubble base, respectively. Based on the recent numerical researches and experimental data, the 3-phase contact line evaporation model seems to be a better alternative than the microlayer evaporation model $[7,8]$.

Moore and Mesler [9] showed experimentally that wall temperature fluctuations can be significant during nucleate boiling. Magrini and Nannei [10] studied the effects of both the wall thickness and the thermal properties of different solids on nucleate boiling. They concluded that both parameters strongly influence nucleation site density and therefore heat transfer. Kenning and Yan [11] used thermochromic liquid crystals (TLCs) to study the temperature field at the backside of a $0.13 \mathrm{~mm}$ thick stainless steel plate. They concluded that the thin wall minimizes lateral heat conduction and, therefore, maximizes the local temperature variation what may be atypical in real boiling situations. Fischer et al. [12] investigated the local temperature distribution under vapor bubbles in reduced gravity. During the bubble growth process, the local cold area was changed with the moving of the contact line, and a distinct local temperature drop about $4 \mathrm{~K}$ can be observed. Similar results were also reported by Jung and Kim [13], as well as by Yabuki et al. [14] who developed a MEMS sensor with high spatio-temporal resolution in temperature measurement of the wall temperature beneath a growing bubble in saturated water. Moghaddam and Kiger [15] also developed a MEMS sensor to measure the continuous bubble boiling of FC-72 under saturation conditions. They indicated that the initial formation of the bubble leads a sudden drop in surface temperature and the observed temperature drop was due to surface cooling resulted from 3-phase contact line evaporation.

Up to now, researches have shown that although the 3-phase contact line region has only micrometer scale in size, the heat transfer in this micro region significantly influences the heat transfer in the bulk region, especially inside the solid wall, and the whole characteristics of heat transfer. The local heat flux reaches a maximum value at the 3-phase contact-line region that can be one order of magnitude or even more higher than the mean input heat flux, and the heat transfer on the micro region can contribute up to $20-30 \%$ of the total heat consumed by a vapor bubble [16]. Obviously, such high heat flux must have extremely effects on the heat conduction inside the solid wall of heater, and the energy supply to the liquid to maintain the nucleate boiling process. The local fluid flow and heat transfer of 3-phase contact line evaporation must be modeled correctly to reveal the mechanism underlying the 3phase contact line heat transfer. In the model proposed by Stephan and Hammer [6], a fourth order differential equation is derived by using the lubrication theory accompanying with the Young-Laplace equation, as well as the kinetic theory of gases with the ClausiusClapeyron equation to model the evaporation rate from the vapor-liquid interface. The equation can be solved to obtain the liquid film thickness, the local heat flux, the position of the contact line, and so on. They showed that the interface of the 3-phase contact line region has a nearly constant slope except the vicinity of the adhesion region where the slope decreases to zero rapidly. Following Stephan and Hammer [6], Dhir and his colleagues [17-19], among many others, simulated numerically single bubble boiling phenomenon with major focus upon bubble behaviors and heat transfer. To avoid burdensome handling of the fourth order differential equation for the 3-phase contact line region, a simplified model with the assumption of a constant interface slope in the 3-phase contact line region was proposed by Son [20] for the planar case, and extended later by Zhao et al. [21] to the axisymmetric one. However, the influence of transient heat conduction inside the solid wall is often ignored for simplicity in the most of studies, in which a constant temperature is adopted for the boundary condition 
instead of the actual solid wall of the heater. Kunkelmann and Stephan [22] performed transient numerical simulations of single bubble pool boiling of HFE-7100 which included the heat transfer both in fluid and in solid phases and between them. The authors showed the spatial variation in surface temperature and heat transfer rates. The heater they simulated is only $0.05 \mathrm{~mm}$ thick, which is even smaller than that used by Kenning and Yan [11]. Thus, the problems pointed by Kenning and Yan [11] still exist. Aktinol and Dhir [23] added a solid heater in their models and compared the results of single bubble pool boiling of water with and without solid wall under the similar conditions. They also compared the results between constant temperature and constant heat flux on the bottom surface of the heater, and pointed out that a constant temperature wall which is artificially fixed a constant superheat on its bottom surface may slightly over-predict (about 7\%) the heat flux from its top surface to the working fluid than a constant heat flux wall, especially for the thin heater. Zhang et al. [24,25] and Zhang [26] numerically studied the influence of heater thermal capability on nucleate pool boiling of saturated water. They also found that the surface temperature of solid wall can vary both temporally and spatially and solid wall thickness and material properties are observed to affect waiting time significantly, and the heater thickness will also affect the surface temperature recovery during nucleate boiling. Additionally, highly conductive materials are able to recover faster than poorly conductive materials. The thickness of solid walls in their simulations is no more than $1 \mathrm{~mm}$, which is still not thick enough to a certain extent.

In the present study, to reveal the transient thermal response of the solid wall on boiling phenomenon, bubble dynamics and heat transfer during single bubble pool boiling of saturated FC-72 are simulated numerically with transient conduction inside the heated solid wall. The Ghost Fluid Method (GFM) is used for the sharp interface representation, while two level set functions are adopted for capturing the liquid-vapor-solid interfaces. Furthermore, as a part of the ground-based advance research of the project of SOBER-SJ10, which is one of the selected experiments of the China space mission SJ-10 [27], more attentions will be paid on the transient thermal response of thick solid wall of the heater, in order for determining a suitable thickness of the heater used in the forthcoming space experiment.

\section{Numerical model}

Following Stephan and Hammer [6], the computational domain for single bubble pool boiling process is divided into micro and

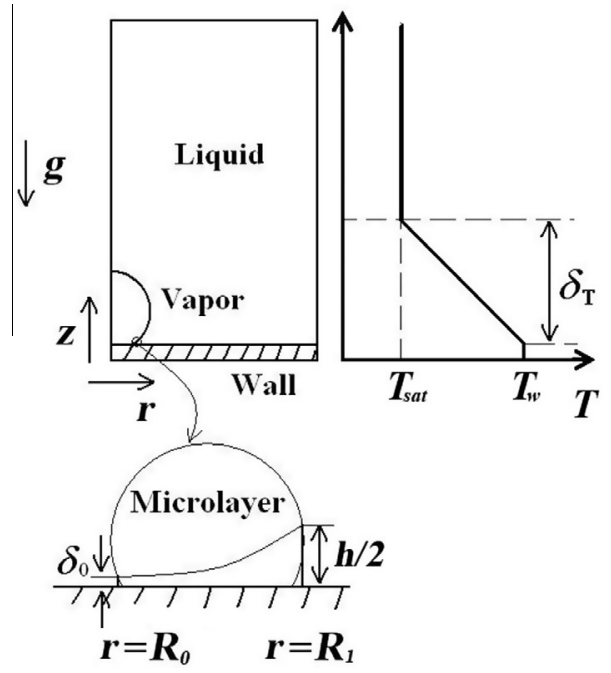

Fig. 1. Computational domain used in the numerical simulation. macro regions (Fig. 1). The following assumptions are made during the present study: (1) the fluid in each phase is Newtonian, viscous and incompressible; (2) the material properties are constant and not influenced by the temperature and pressure; (3) the flow in fluid phases is axisymmetric and laminar, while the heat conduction inside the solid wall is also axisymmetric; (4) the apparent contact angle is constant; (5) a constant temperature is maintained on the bottom of the solid wall.

The computation model is described in a cylindrical coordinate. The mass, momentum, and energy conservation equations for the macro region are written as

$\nabla \cdot \mathbf{u}=0, \quad \Omega=\left\{\Omega_{v} \cup \Omega_{l}\right\}$

$\mathbf{u}=0, \quad \Omega_{s}$

$\rho\left(\frac{\partial \mathbf{u}}{\partial t}+\mathbf{u} \cdot \nabla \mathbf{u}\right)=-\nabla p+\nabla \cdot \mu\left(\nabla \mathbf{u}+\nabla \mathbf{u}^{T}\right)+\rho\left[1-\beta_{T}\left(T-T_{\text {sat }}\right)\right] \mathrm{g}$,

$\Omega=\left\{\Omega_{v} \cup \Omega_{l}\right\}$

$\begin{cases}\frac{\partial T}{\partial t}+\mathbf{u} \cdot \nabla T=\frac{1}{\rho c_{p}} \nabla \cdot k(\nabla T), & \Omega=\left\{\Omega_{l} \cup \Omega_{s}\right\} \\ T=T_{s a t}\left(p_{v}\right), & \Omega_{v}\end{cases}$

The conservation equations are solved accompanied with the jump conditions [28] at the interface

$[\mathbf{u}]_{\Gamma}= \begin{cases}-[1 / \rho]_{\Gamma} \dot{m} \mathbf{n}, & \Gamma_{l v} \\ 0, & \Gamma_{f s}\end{cases}$

$[\mu(\nabla u \cdot \mathbf{n}, \nabla v \cdot \mathbf{n}) \cdot \mathbf{t}+\mu(\nabla u \cdot \mathbf{t}, \nabla v \cdot \mathbf{t}) \cdot \mathbf{n}]_{\Gamma}=0, \quad \Gamma_{l v}$

$[p]_{\Gamma}=2[\mu(\nabla u \cdot \mathbf{n}, \nabla v \cdot \mathbf{n}) \cdot \mathbf{n}]_{\Gamma}-\sigma \kappa-[1 / \rho]_{\Gamma} \dot{m}^{2}, \quad \Gamma_{l v}$

$T_{l}=T_{v}=T_{s a t}, \quad \Gamma_{l v}$

$[k \nabla T \cdot \mathbf{n}]_{\Gamma}= \begin{cases}\dot{m} h_{f g}, & \Gamma_{l v} \\ 0, & \Gamma_{f s}\end{cases}$

where the jump across the interface is defined as $[\Lambda]_{\Gamma}=\Lambda_{l}-\Lambda_{v}$ for $\Gamma_{l v}$ and $[\Lambda]_{\Gamma}=\Lambda_{f}-\Lambda_{s}$ for $\Gamma_{f s}$, and the mass flux $\dot{m}$ is defined as $\dot{m}=\rho\left(\mathbf{u}_{\text {int }}-\mathbf{u}\right) \cdot \mathbf{n}$.

In the present paper, the assumption of constant interface slope for the axisymmetric micro region [20] is adopted, namely

$\frac{d \delta}{d r}=\tan \gamma$

where $\gamma$ denotes the apparent contact angle. Thus, based on the combination of the mass, momentum, and energy equations for the micro region, formulations for the area averaged heat flux $q_{\text {mic }}$ and mass flux $\dot{m}_{\text {mic }}$ of the micro region is obtained, respectively [21]

$q_{\text {mic }}=\frac{2 \pi k_{l}\left(T_{w}-T_{\text {sat }}\right)}{\Delta A_{\text {mic }}}\left[\begin{array}{c}\left(R_{1}-\frac{h}{2 \tan \gamma}\right) \frac{1}{\tan \gamma} \ln \left(\frac{h}{2} \frac{h_{e V}}{k_{l}}+1\right) \\ +\frac{h}{2 \tan ^{2} \gamma}-\frac{k_{l}}{h_{e V}} \frac{1}{\tan ^{2} \gamma} \ln \left(\frac{h}{2} \frac{h_{e V}}{k_{l}}+1\right)\end{array}\right]$

$\dot{m}_{m i c}=\frac{q_{m i c}}{h_{f g}}$

Two level set functions are used for capturing the liquidvapor-solid interfaces. Firstly, the liquid-vapor interface is advanced by solving the following equation

$\frac{\partial \varphi}{\partial t}=-\mathbf{u}_{\text {int }} \cdot \nabla \varphi$

where $\mathbf{u}_{\text {int }}$ is the interface velocity, which is defined as

$\mathbf{u}_{\text {int }}=\frac{\dot{m} \mathbf{n}}{\rho}+\mathbf{u}$ 
Re-initialization is needed to maintain a signed distance function from the interface

$\varphi_{\tau}+S\left(\varphi_{0}\right)(1-|\varphi|)=0$

where the smoothed out sign function $S\left(\varphi_{0}\right)=\frac{\varphi_{0}}{\sqrt{\varphi_{0}^{2}+\Delta x^{2}}}$, and $\varphi_{0}$ is the solution of Eq. (13).

Another level set function $\psi$ is also used to treat the immersed solid surface which is defined as a signed distance from the fixed fluid-solid interface. The level set function $\psi$ is not necessary to be advanced.

The material properties of different phases are described as

$\rho=\rho_{s}+\left(\rho_{v}+\left(\rho_{l}-\rho_{v}\right) H(\varphi)-\rho_{s}\right) H(\psi)$

$\mu=\mu_{s}+\left(\mu_{v}+\left(\mu_{l}-\mu_{v}\right) H(\varphi)-\mu_{s}\right) H(\psi)$

$k^{-1}=k_{s}^{-1}+\left(k_{l}^{-1} H(\varphi)-k_{s}^{-1}\right) H(\psi)$

where $H$ is the discontinuous Heaviside function, which is described as

$H= \begin{cases}1 & \text { if } \varphi \text { or } \psi>0 \\ 0 & \text { if } \varphi \text { or } \psi \leqslant 0\end{cases}$

In order for that the process of bubble growth is not affected by the computational boundary, the computational domain of the fluid was chosen to be $(1 L, 2 L)$. Here, $L=\sqrt{\sigma /\left[\left(\rho_{l}-\rho_{v}\right) g\right]}$ is the Laplace length. Experimental observations, as well as mechanism models such as that by Mikic and Rohsenhow [29], show that the disturbed region on the heated surface caused by a growing bubble is roughly 2 times the departure diameter, which is of the same order of magnitude of the Laplace length $L$. Furthermore, making reference to others, for example, Son and Dhir [17] among many others, a radial size of the computational domain of $1 \mathrm{~L}$. By considering the axisymmetric domain, it implies a disturbed region of $2 L$, satisfying the necessary condition for a bubble isolated from its neighbors. Verification can be found in Zhang [26].

The boundary conditions used in the present simulation are as follows.

At the axis of symmetry,

$u=\frac{\partial v}{\partial r}=\frac{\partial T}{\partial r}=\frac{\partial \varphi}{\partial r}=0$

At the right of domain,

$u=\frac{\partial v}{\partial r}=\frac{\partial T}{\partial r}=\frac{\partial \varphi}{\partial r}=0$

At the bottom of the wall,

$u=v=0, \quad T=T_{w}$

At the top boundary,

$\frac{\partial u}{\partial z}=\frac{\partial v}{\partial z}=\frac{\partial \varphi}{\partial z}=0, \quad T=T_{s a t}$

Another two conditions in the vicinity of three-phase contact line inside the wall also need to be considered

$\left(k \frac{\partial T}{\partial z}\right)_{m i c}=-q_{m i c}, \quad \frac{\partial \varphi}{\partial z}=-\cos \gamma$

Initial still flow field is used as the initial velocity condition, namely

$u=v=0$ at $t=0$

While the initial thermal distribution is given as shown in Fig. 1, where the initial thermal boundary-layer thickness is determined by the correlation [30]

$\delta_{T}=7.14\left(\frac{v_{l} \alpha_{l}}{g \beta_{T} \Delta T}\right)^{1 / 3}$
A small truncated sphere bubble with the initial radius of $0.05 \mathrm{~L}$ is set on the top surface of the solid wall at the beginning of a bubble cycle. Constant nucleating superheat corresponding to the given cavity diameter, $D_{c}$, of the nucleate site [31]

$\Delta T_{n}=T_{w}-T_{s a t}=\frac{4 f_{1} \sigma T_{s a t}}{D_{c} h_{f g} \rho_{v}} /\left(1-\frac{f_{2} D_{c}}{2 f_{1} \delta_{T}}\right)$

is chosen as the criterion for determining the beginning of the subsequent bubble cycle, where $f_{1}$ and $f_{2}$ are functions of the contact angle. The characteristic parameters, such as the waiting time, the growth time, and so on, may be strongly influenced by the cavity size $D_{c}$. The spatio-temporal averaged superheat and the corresponding spatio-temporal averaged heat flux increase slightly with the decrease of the cavity size. The global characteristic, or the boiling curve, however, is not sensitive to that quantity. Detailed discussion on this question can be found in Zhang [26].

The primary variables is non-dimensioned by the Laplace length $L$ and the characteristic velocity $U=\sqrt{g L}$, and the dimensionless temperature is defined as $\theta=\frac{T-T_{\text {sat }}}{T_{w}-T_{\text {sat }}}$.

A standard MAC grid is used for the spatial discretization where the velocities are defined at the grid surfaces and other variables are defined at the grid nodes. The projection method is used to solve numerically the Navier-Stokes equations. The 2nd order ENO scheme [32] is used for the discretization of the convection term in the momentum and energy equations, and the central difference is used for the diffusion terms. For more details about the related solving procedure and algorithm, one can see Zhang [26] and Zhao et al. [33,34]. To save the computing time without losing the accuracy of numerical results, the mesh number used in the computations of this study is $150 \times 300$. Detailed analysis on the mesh dependency can be found in our previous works $[21,26]$.

Furthermore, multi-cycle simulations of bubble growth process are carried out for all cases below to eliminate the influence of unreal initial conditions.

\section{Results and discussion}

The working fluid used in the present study is saturated FC-72 at $0.1 \mathrm{MPa}$. The heater material is $\mathrm{SiO}_{2}$ and its properties are listed in Table 1 . A constant apparent contact angel of $42^{\circ}$ is used in all cases. This value is nearly the middle of those applied by Sielaff et al. [35], in which values of $50^{\circ}$ as well as $30^{\circ}$ were applied for the calculations for FC-72 at $0.05,0.07$, and $0.09 \mathrm{MPa}$. The influence of the apparent contact angle on bubble dynamics and heat transfer is beyond the research scope of the present study, and then is not included here.

Table 2 summarizes the conditions and results of the present simulation on single bubble pool boiling. A constant temperature boundary condition is fixed on the bottom surface of solid walls. The difference between this fixed temperature and the saturate temperature of FC-72 at $0.1 \mathrm{MPa}$ will be referred as the nominal superheat. Thus, both the spatio-temporal averaged heat flux $q_{w, a v e}$ and the spatio-temporal averaged superheat $\Delta T_{w, \text { ave }}$ on the top surface of the solid wall, which are averaged over the whole top surface through a whole bubble growth cycle, are not controllable variables but dependent ones. For the sake of clarity, only the

Table 1

Material properties of solid wall (fused silica $\mathrm{SiO}_{2}$ ).

\begin{tabular}{ll}
\hline Density $\rho\left(\mathrm{kg} / \mathrm{m}^{3}\right)$ & 2220 \\
Specific heat $C_{p}(\mathrm{~J} / \mathrm{kg} \cdot \mathrm{K})$ & 865 \\
Thermal conductivity $k(\mathrm{~W} / \mathrm{m} \cdot \mathrm{K})$ & 1.4775 \\
Thermal diffusivity $\alpha\left(\times 10^{-6} \mathrm{~m}^{2} / \mathrm{s}\right)$ & 0.77 \\
\hline
\end{tabular}


Table 2

Simulation conditions of single bubble pool boiling in different cases.

\begin{tabular}{llllll}
\hline Case No. & $h_{w}(\mathrm{~mm})$ & $\Delta T_{b}(\mathrm{~K})$ & $D_{c}(\mathrm{~m})$ & $\Delta T_{w, \text { ave }}(\mathrm{K})$ & $q_{w, \text { ave }}\left(\mathrm{W} / \mathrm{cm}^{2}\right)$ \\
\hline 1 & 1.0 & 10.0 & $1 \times 10^{-6}$ & 7.24 & 0.52 \\
2 & 1.0 & 7.0 & $1 \times 10^{-6}$ & 4.8 & 0.23 \\
3 & 1.0 & 15.0 & $1 \times 10^{-6}$ & 12.1 & 0.96 \\
4 & 5.0 & 10.0 & $1 \times 10^{-6}$ & 6.3 & 0.41 \\
\hline
\end{tabular}

steady values of $q_{w, a v e}$ and $\Delta T_{w, a v e}$ during the last cycle for each case are listed in Table 2.

Fig. 2 shows a typical multi-cycle process of a single bubble growing on $\mathrm{SiO}_{2}$ heater with thickness of $1 \mathrm{~mm}$ under the nominal superheat of $10 \mathrm{~K}$ (Case 1 ). In the figure, we can see that the bubble growth process changes from one cycle to another one in the early stage. The main reason for this uncertain process is the ideal but non-physical initial condition specified in the numerical simulation. Therefore, to eliminate the influence of the initial condition on the process of bubble growth and then on the heat transfer of pool boiling, multi-cycle simulation is necessary. It can be seen in Fig. 2 that bubble growth processes approach almost the same during the last several cycles, which indicates the achievement of steady state condition. Parameters such as bubble growth time $t_{g}$, waiting time $t_{w}$, departure diameter $D_{d}$, nucleation superheat $\Delta T_{n}$, spatio-temporal averaged heat flux $q_{w, \text { ave }}$ and spatio-temporal averaged surface superheat $\Delta T_{w, \text { ave }}$ on the top surface of the solid wall also reach steady values at the same time (Fig. 3). For the sake of briefness, only the results for cycles with the cycle number greater than 46 are shown in Fig. 2. Similar steady states can also be achieved in Cases 2 and 3. In Case 4, the state cannot reach steady state, which will be discussed in detail in the following section.

Fig. 4 shows the simulated boiling curve $q_{w, a v e}$ vs $\Delta T_{w, \text { ave }}$ predicted by the present numerical simulation. The experimental data obtained by Moghaddam and Kiger [15] (represented by M and K in the figure) and the prediction of the correlation by Zuber [36] are also shown for comparison. Only the ultimate steady results of Cases 1, 2, and 3, in which the heater thickness is $1 \mathrm{~mm}$ and steady states can be achieved, are plotted in Fig. 4. Generally, the numerical results are in good agreement with the experimental data and the prediction of the correlation by Zuber [36].

Based on the simulations with a $0.5 \mathrm{~mm}$ thick stainless steel heater, Aktinol and Dhir [23] pointed out that a constant temperature boundary condition artificially fixed on the bottom surface may slightly over-predict the heat flux from its top surface to the working fluid than a constant heat flux one. Although thicker heaters of a different kind of material are used in the present simulation, it is still necessary to analyze the influence of the thickness of heater on the nucleate boiling.
Fig. 5 shows the calculated multi-cycle process of Case 4, while variations of bubble growth time $t_{g}$, waiting time $t_{w}$, departure diameter $D_{d}$, nucleation superheat $\Delta T_{n}$, spatio-temporal averaged heat flux $q_{w, a v e}$ and spatio-temporal averaged superheat $\Delta T_{w, a v e}$ with bubble cycles are shown in Fig. 6. For the sake of briefness, only the results for cycles with the cycle number greater than 36 are shown in Fig. 6. As shown in Fig. 5, bubble growth processes are different cycle by cycle not only in the early stage but also in the later. Thus, Case 4 failed to reach a steady state during the whole simulation of the 54 cycles. Bubble growth time, waiting time and departure diameter also evolved irregularly as shown in Fig. 6.

The differences between the cases of two thicknesses can be explained by the thermal penetration depth. According to heat transfer theory, the thermal penetration depth of a semi-infinite solid can be determined by Eq. (28)

$$
\delta_{h}=\sqrt{\pi \alpha_{h} t}
$$

If the thermal penetration depth is larger than the heater thickness, the temperature distribution in the heater will be approximately linear distribution, especially in the outer boundary of the solid wall. Otherwise, the temperature distribution in the lower region of the heater will approximately maintain the initial value, namely the disturbance of temperature on the top surface by bubbling process has not enough time to propagate throughout the whole solid wall. Indubitably, the simulation of bubble growth process can reach a steady state only after the heated solid wall is thermal-penetrated.

In Figs. 7 and 8, the temperature distributions at bubble departure and at bubble nucleation along longitudinal direction inside solid wall under the same nominal superheat of $10 \mathrm{~K}$ are plotted for Cases 1 and 4, respectively. Five locations along the radial direction are selected in each case to ensure the representative of these temperature distributions.

The calculated physical time is $0.74 \mathrm{~s}$ in the case of $1 \mathrm{~mm}$ heater, and the corresponding thermal penetration depth is $1.34 \mathrm{~mm}$, which is larger than the heater thickness. Correspondingly, the temperature distribution in the outer boundary of the heater is approximately linear distribution (Fig. 7), which means the heater is thermal-penetrated in this cycle. On the contrary, although the calculated physical time of boiling process in the case of $5 \mathrm{~mm}$ heater was approximately $1.35 \mathrm{~s}$, which is more than that in Case 1 , the thermal penetration depth $\delta_{h}$ of about $1.8 \mathrm{~mm}$ was determined by Eq. (28), which is much smaller than the heater thickness. This is also consistent with the results shown in Fig. 8. The solid temperature below the depth of about $2.0 \mathrm{~mm}$ is almost equal to the initial value, implying energy supply for bubbling process on its top surface is mainly provided by those stored inside the upper

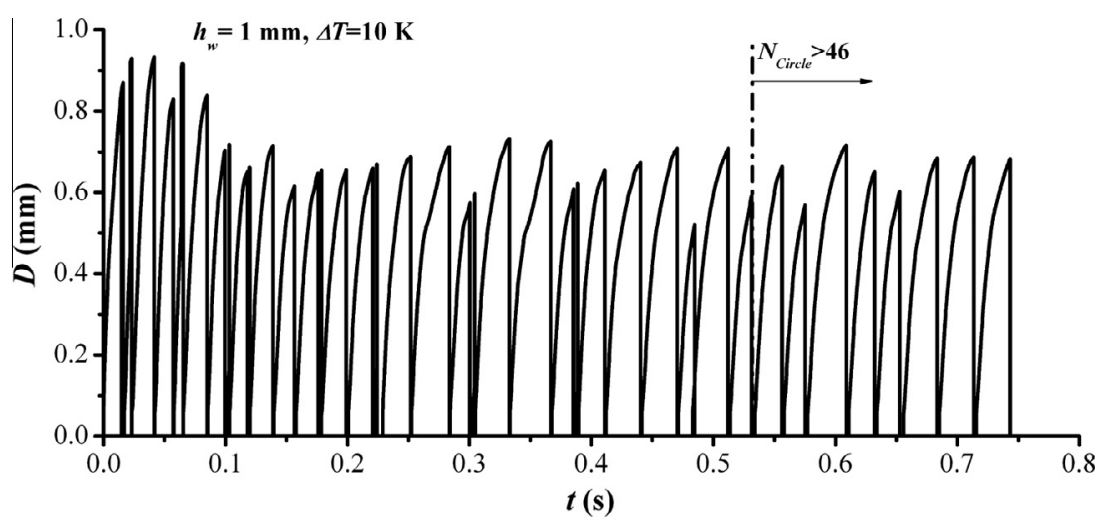

Fig. 2. Multi-cycle process of bubble growth in Case 1 . 

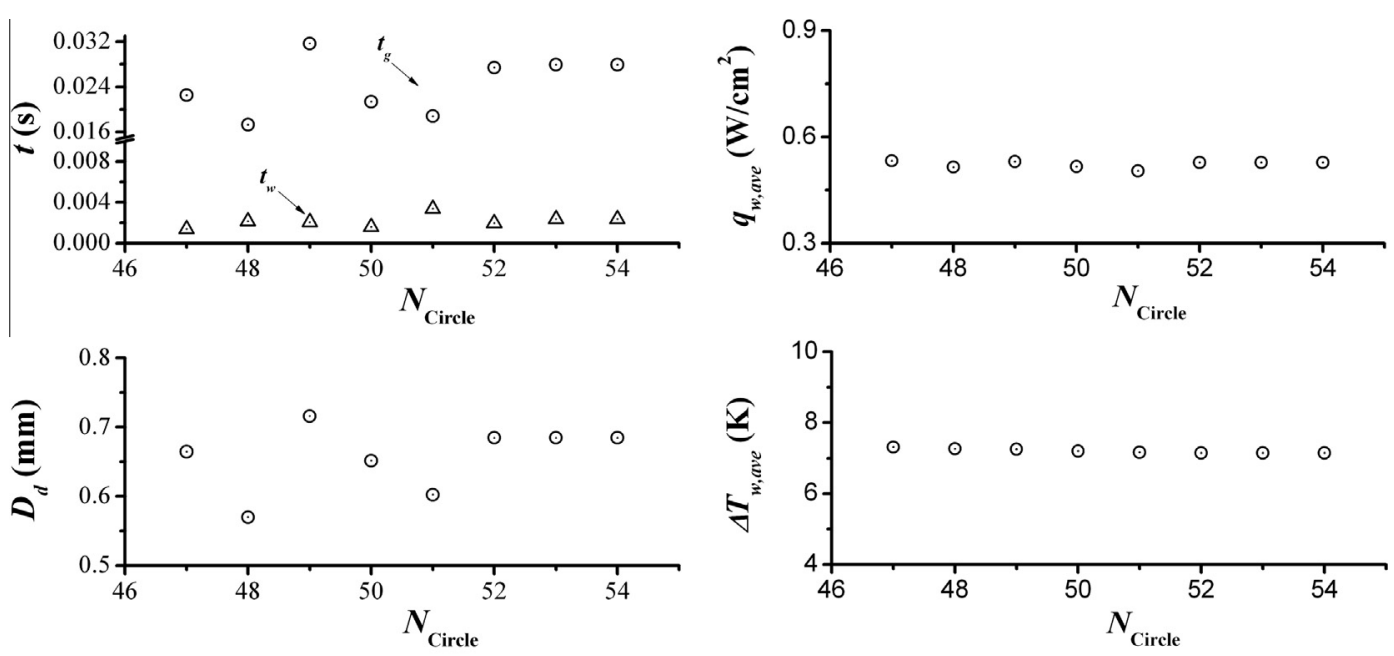

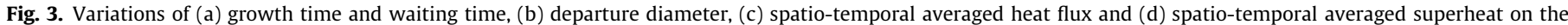
heater's surface for cycles 37-54 in Case 1.

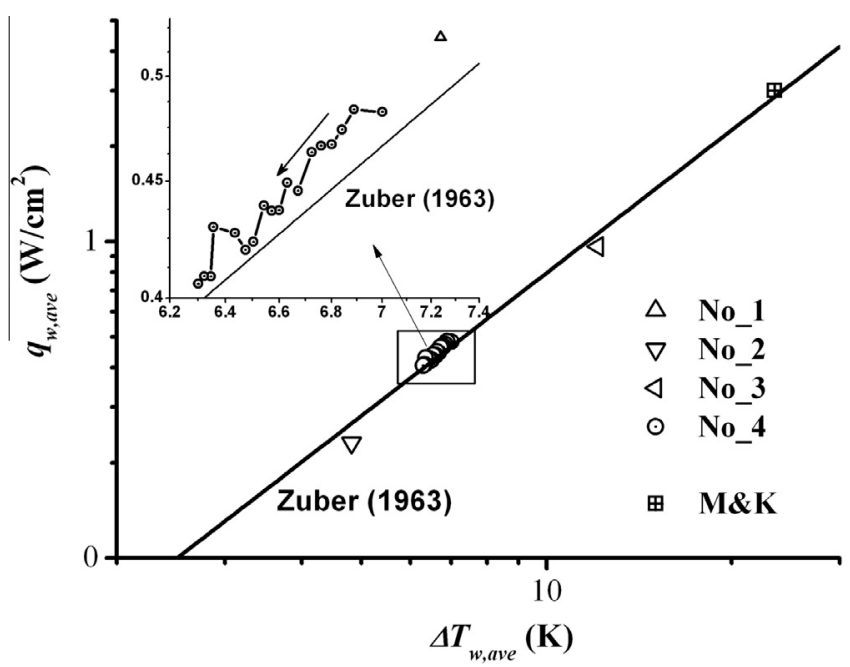

Fig. 4. Comparison of simulation results with experimental data and the prediction by the correlation by Zuber [36].

part of the solid wall, just like that in a transient quenching process.

Then, in Case 4 with the thickness of $5 \mathrm{~mm}$, due to the heater is not thermal-penetrated throughout the simulation, the temperature field inside the solid wall cannot reach a steady state, so that the spatio-temporal averaged heat flux and superheat on its top surface cannot reach a steady state, too. In fact, they become lower and lower as shown in Fig. 6.

In accordance with the common knowledge [37-39], however, if the rate of unsteady factors' change is small enough, and the characteristics of the unstable process are approximatively in good agreement with those in the steady state, the quasi-steady state will be achieved in the process. As shown in Fig. 4, the spatio-temporal averaged heat flux and the corresponding spatio-temporal averaged superheat on the top surface of solid wall in Case 4 (cycle number $>36$ ) are approximatively in good agreement with the present simulations of steady state of single bubble pool boiling, the experimental data of Moghaddam and Kiger [15], and the prediction of the correlation by Zuber [36]. Thus, the achievement of quasi-steady state of single bubble pool boiling can be concluded in Case 4. Furthermore, according to Eq. (28), one can derive that the change of the thermal penetration depth, $\Delta \delta_{h}$, can be expressed as

$\frac{\Delta \delta_{h}}{\delta_{h}}=\frac{1}{2} \frac{t_{c}}{t}$

Then, for cycles near the 50th one, $\Delta \delta_{h} / \delta_{h} \approx 1.8 \%$, which is much smaller than the thermal penetration depth at the same time, and thus the thermal condition far enough away from the nucleation site inside the solid wall can be considered as in steady state, which will then be satisfied with the necessary requirement of steady state of boiling process. So, although the spatio-temporal

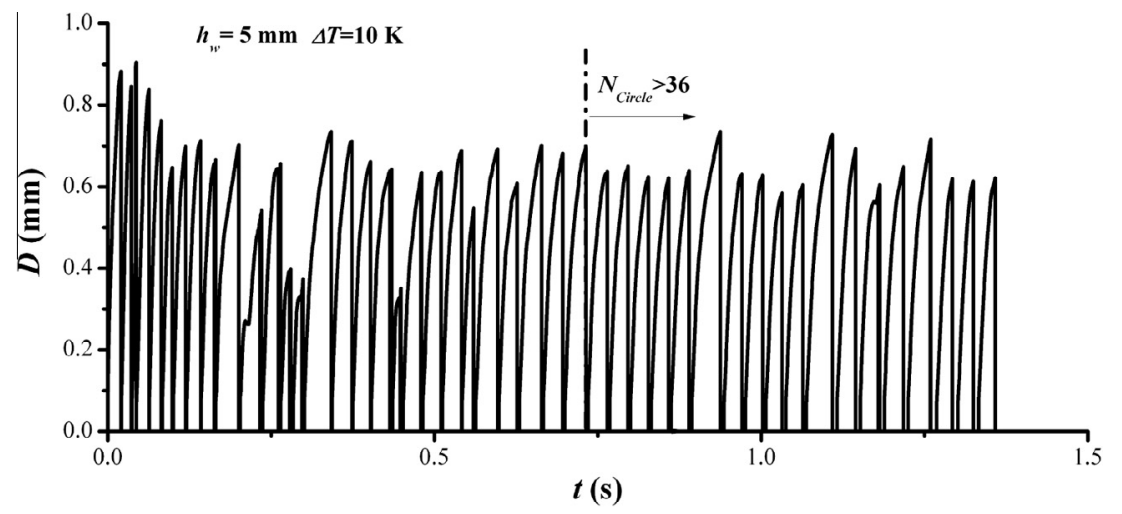

Fig. 5. Multi-cycle process of bubble growth in Case 4 . 

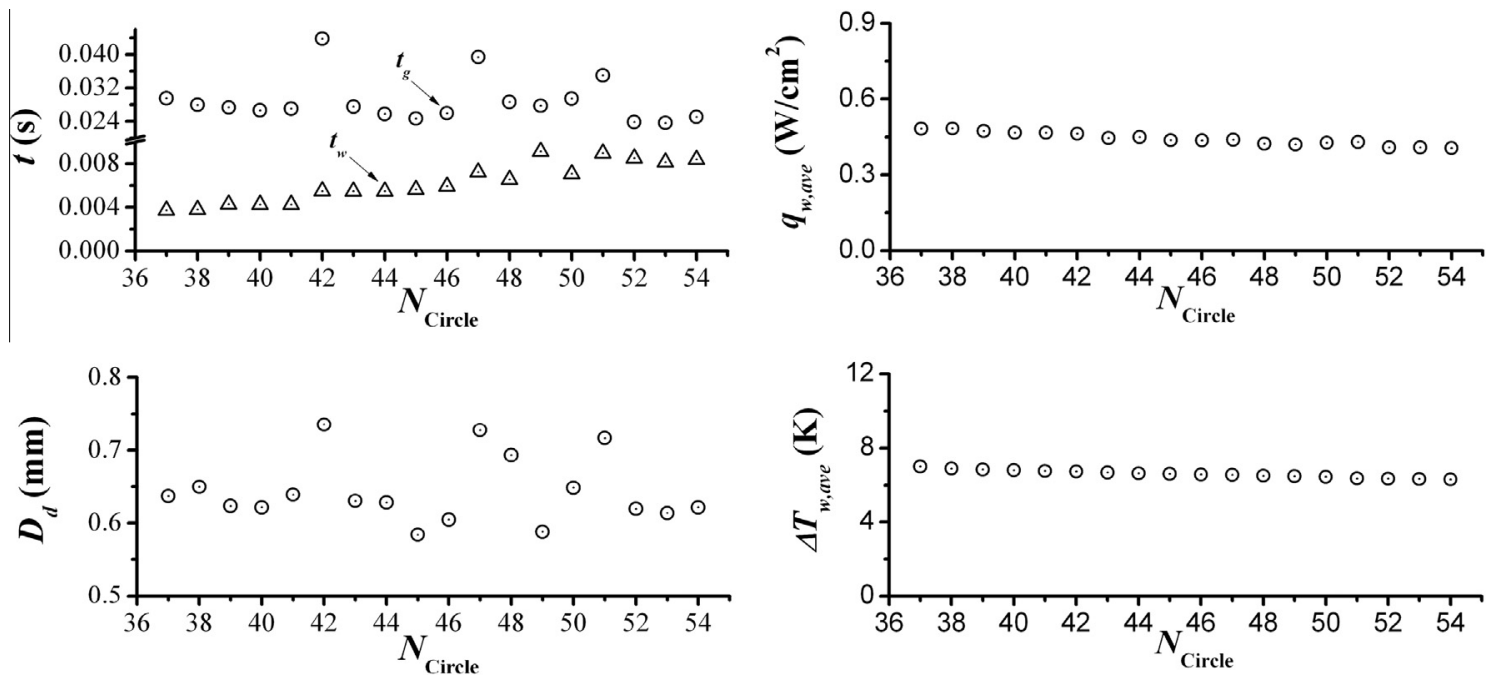

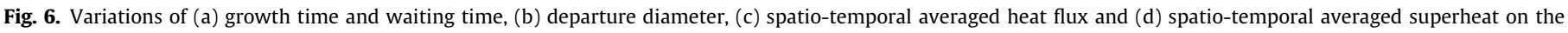
heater's surface for cycles $47-54$ in Case 4.
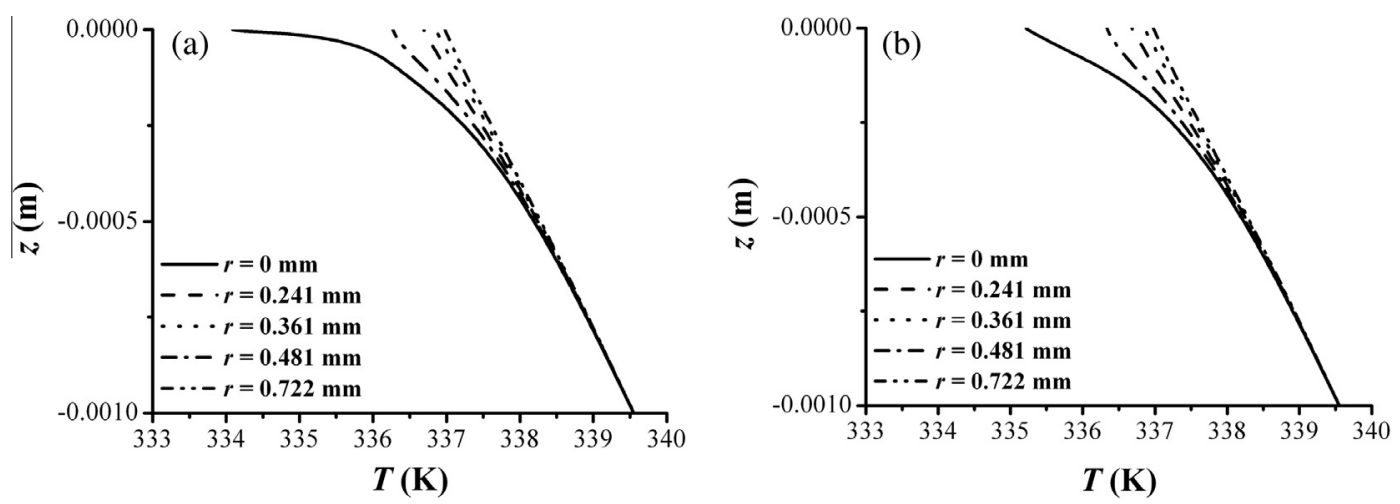

Fig. 7. Temperature distributions along longitudinal direction at (a) departure and (b) nucleation on the axis in Case 1.
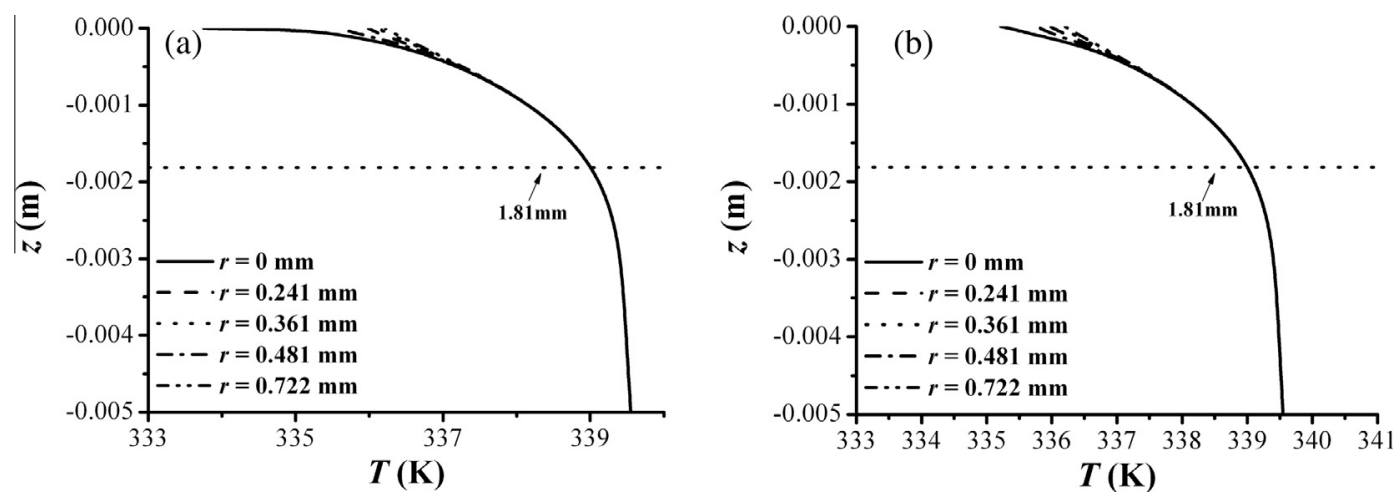

Fig. 8. Temperature distributions along longitudinal direction at (a) departure and (b) nucleation on the axis in Case 4.

averaged heat flux and superheat will become lower and lower as computation goes on continually in Case 4, each calculated point is still represent a quasi-steady status, and the whole computation depicts a quasi-steady process until it reach the ultimate steady statue (if computational time is long enough). In other words, for a thicker heater, it will go through a long-period quasi-steady process to reach a steady statue in numerical simulation, which will cost a lot of computational time. Fortunately, these intermediate processes are not entirely without significance. They can depict a quasi-steady process like quenching, even provide a continuous boiling curve as those obtained in quasi-steady boiling experiments [37-39].

Fig. 9 shows the temperature distributions on the heating surface at five different time in a typical bubble growth cycle for both cases. It is obvious that a sharp temperature drop can be observed clearly near the contact-line region during the bubble growth process because of the large evaporation effect in the contact-line region. The temperature drop area moves with the movement of contact line. After the bubble detached, the temperature drop disappears gradually and the temperature in the area affected by the 

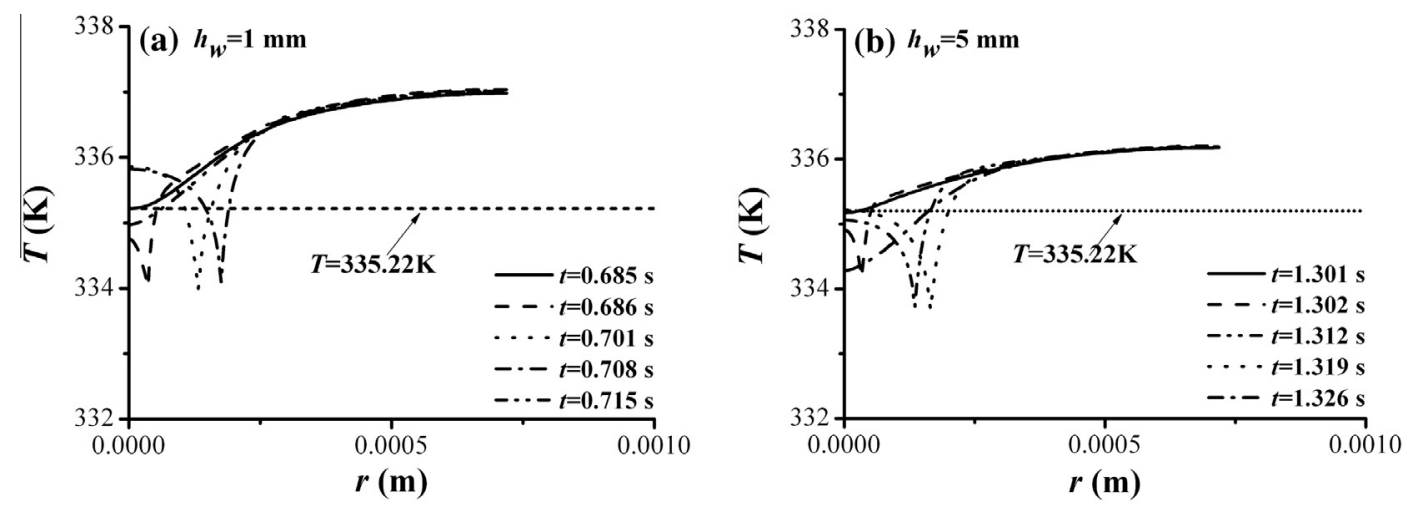

Fig. 9. The evolutions of temperature distribution on heating surface. (a) in Case 1, (b) in Case 4.
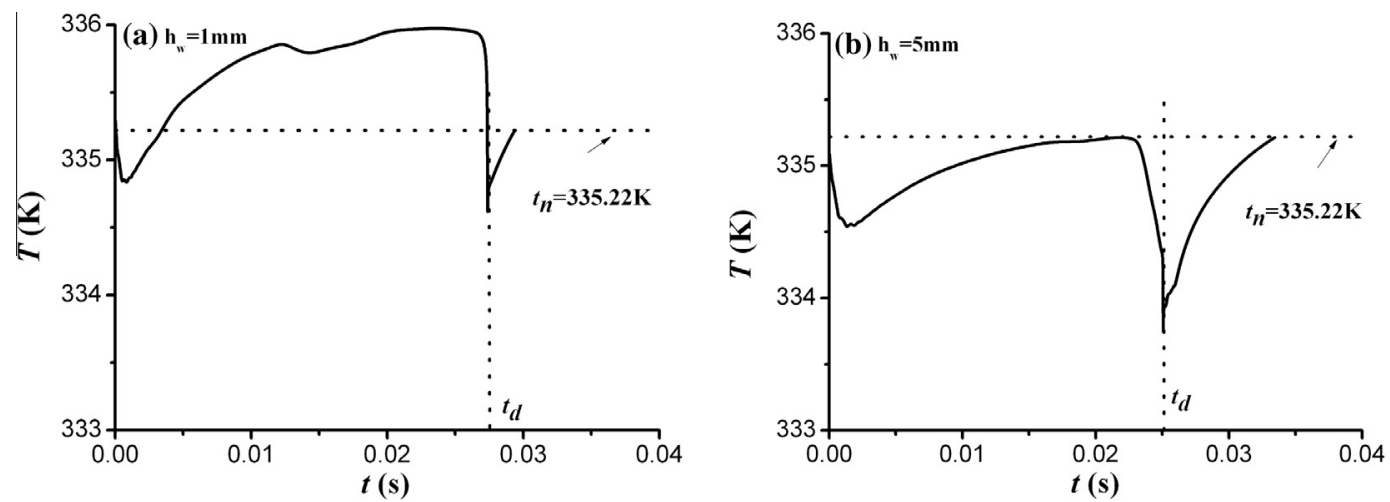

Fig. 10. The evolutions of temperature at the nucleate site. (a) in Case 1, (b) in Case 4.
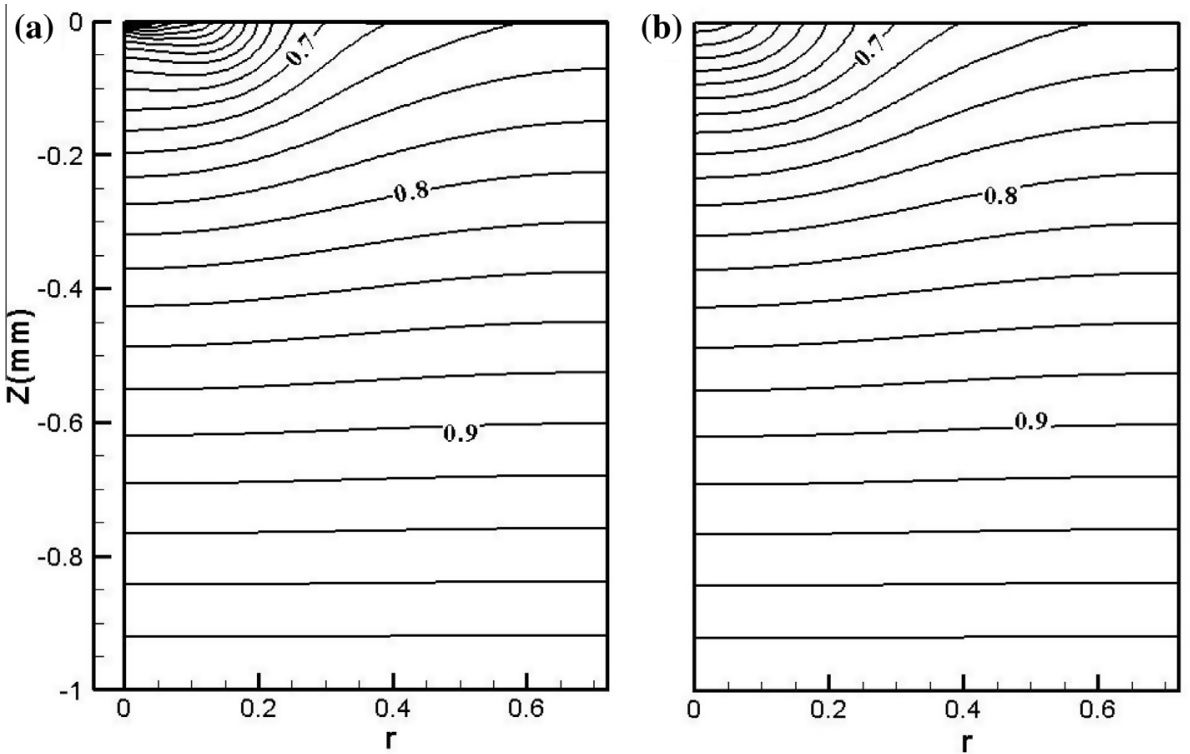

Fig. 11. Temperature distributions in the heater at (a) departure and (b) nucleation in Case 1.

movement of contact line begins to rise up because of the transient conduction of heater.

In Fig. 10, the changes of temperature at the nucleation site on the top surface of solid walls in both cases are shown. Twice temperature drops occur in both curves. The first drop is smaller and lasts for a very short period. The first fact is not coincident with experimental observations. The reason rests on the simplified assumption in the present model. In the present model, as well as in most other models for computing boiling process, instead of the actual nucleation process, a small truncated spherical bubble is located artificially on the nucleation site for setting an initial bubble surface. Then violent heat absorbing from the surroundings in the inertia-controlled stage of the initial bubble growth cannot be simulated. Small amount of heat is absorbed to maintain the 


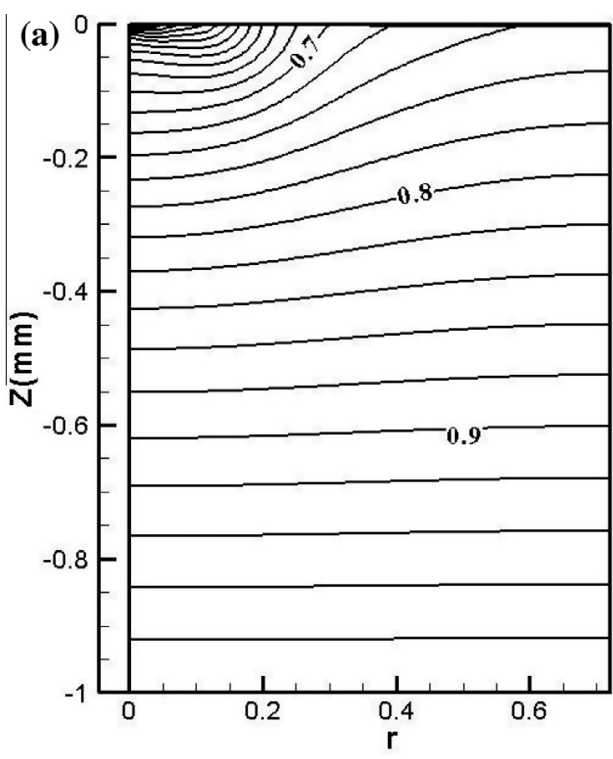

(b)

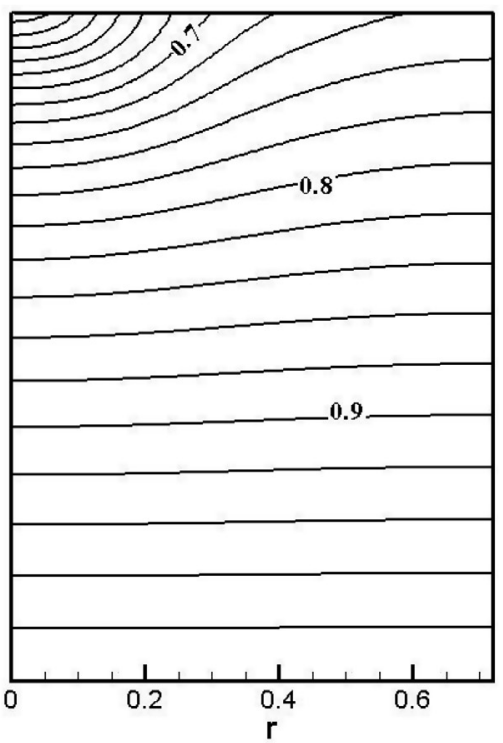

Fig. 12. Temperature distributions in the heater at (a) departure and (b) nucleation in Case 4.

growth of the initial small bubble, and thus only a smaller temperature drop at the nucleate site is observed here.

After the contact line passes by, the bubble base extends and a local dry-spot emerges along with the bubble growth. The nucleate site is heated again, resulting in the temperature increase. The heating stage will be ended when the contact line extends to its maximal position and then recedes. Liquid re-floods on the local dry-spot with the receding of the contact line, and thus, a second temperature drop occurs. After bubble departure, the temperature rises again until the nucleate criterion reaches and then a new bubble growth cycle begins.

In Figs. 11 and 12, the temperature distributions in the heater at bubble departure and at bubble nucleation are plotted for Cases 1 and 4 , respectively. Because the isotherms in the section of below $1 \mathrm{~mm}$ of the heater are almost horizontal in Case 4, the isotherms in that region are not shown in Fig. 12. It can be seen that both of the thermal penetration depths caused by the processes of bubble growth and departure in the two cases are almost $0.5 \mathrm{~mm}$. This is in good agreement with the result of Fischer et al. [12], in which a thermal penetration depth of $0.5 \mathrm{~mm}$ was reported for single bubble boiling of FC-72 on CaF heater with the thickness of $2 \mathrm{~mm}$. Furthermore, it's also in good agreement qualitatively with that of Jung and Kim [13], in which a thermal penetration depth of $1 \mathrm{~mm}$ was reported for single bubble boiling of water on CaF heater with the thickness of $10 \mathrm{~mm}$. The results reported in the two latter cases were based on their experimental measurements on the temperature distribution and the corresponding numerical simulations. Thus, the suitable thickness for the heater used in the SOBER-SJ10 experiment aboard the Chinese recoverable satellite SJ-10 should be approximately $2 \mathrm{~mm}$.

\section{Conclusions}

Using the Ghost Fluid Method for sharp interface representation, the complete single bubble pool boiling processes including the transient thermal response of the solid wall are numerically simulated. In the simulation, both the spatio-temporal averaged heat flux and superheat on the top surface of solid wall are not controllable but dependent variables.

The wall thickness strongly influences the bubble growth process. The $5 \mathrm{~mm}$-thick heater failed to reach a steady state in the present simulation due to much limited computational time, while in all cases of the $1 \mathrm{~mm}$-thick heater steady states can be reached because of the smaller thickness needed to be thermal-penetrated.

The calculated spatio-temporal averaged heat flux and superheat of 1 - and $5 \mathrm{~mm}$-thick heaters are both in good agreement with the prediction of the correlation by Zuber [36] and the experimental data of Moghaddam and Kiger [15]. Thus, the achievement of quasi-steady state of single bubble pool boiling can be concluded in Case 4. Furthermore, for a thicker heater, it will go through a long period quasi-steady process to reach a steady statue (if computational time is long enough). These intermediate processes, however, can also provide meaningful information on boiling process, even provide a continuous boiling curve as those obtained in quasi-steady boiling experiments

A clear sharp temperature drop is produced inside solid wall under the bubble base because of the large evaporation effect in the contact line region. The temperature drop area moves with the movement of contact line and after bubble detached, the temperature in the area affected by the movement of contact line begins to rise up because of the transient conduction of heater.

Based on the analysis of the thermal penetration depths caused by the processes of bubble growth and departure, a suitable thickness of about $2 \mathrm{~mm}$ is proposed for the heater used in the SOBERSJ10 experiment aboard the Chinese recoverable satellite SJ-10.

\section{Conflict of interest}

None declared.

\section{Acknowledgments}

The present study is supported financially by the National Natural Science Foundation of China under the grants of 11372327 and 11402273, and the Strategic Priority Research Program on Space Science, the Chinese Academy of Sciences under the grant of XDA04020404.

\section{References}

[1] H.K. Forster, N. Zuber, Dynamics of vapor bubbles and boiling heat transfer, AIChE J. 1 (1955) 531-535.

[2] S.I. Haider, R.L. Webb, A transient micro-convection model of nucleate pool boiling, Int. J. Heat Mass Transfer 40 (1997) 3675-3688. 
[3] C.Y. Han, P. Griffith, The mechanism of heat transfer in nucleate pool boiling part II, the heat flux-temperature difference relation, Int. J. Heat Mass Transfer 8 (1965) 905-914.

[4] C.Y. Han, P. Griffith, The mechanism of heat transfer in nucleate pool boiling part I, bubble initiation, growth and departure, Int. J. Heat Mass Transfer 8 (1965) 887-904.

[5] M.G. Cooper, A.J.P. Lloyd, The microlayer in nucleate pool boiling, Int. J. Heat Mass Transfer 12 (1969) 915-933.

[6] P. Stephan, J. Hammer, A new model for nucleate boiling heat transfer, Heat Mass Transfer 30 (1994) 119-125.

[7] P. Stephan, J. Kern, Evaluation of heat and mass transfer phenomena in nucleate boiling, Int. J. Heat Fluid Flow 25 (2004) 140-148.

[8] J. Kim, Review of nucleate pool boiling bubble heat transfer mechanisms, Int. J. Multiphase Flow 35 (2009) 1067-1076.

[9] F.D. Moore, R.B. Mesler, The measurement of rapid surface temperature fluctuations during nucleate boiling of water, AIChE J. 7 (1961) 620-624.

[10] U. Magrini, E. Nannel, On the influence of the thickness and thermal properties of heating walls on the heat transfer coefficients in nucleate pool boiling, J. Heat Transfer 97 (2) (1975) 173-178.

[11] D.B.R. Kenning, Y. Yan, Pool boiling heat transfer on a thin plate: features revealed by liquid crystal thermography, Int. J. Heat Mass Transfer 39 (15) (1996) 3117-3137.

[12] S. Fischer, S. Herbert, A. Sielaff, E.M. Slomski, P. Stephan, M. Oechsner, Experimental investigation of nucleate boiling on a thermal capacitive heater under variable gravity conditions, Microgravity Sci. Technol. 24 (3) (2012) 139-146.

[13] S. Jung, H. Kim, An experimental method to simultaneously measure the dynamics and heat transfer associated with a single bubble during nucleate boiling on a horizontal surface, Int. J. Heat Mass Transfer 73 (2014) 365-375.

[14] T. Yabuki, T. Hamaguchi, O. Nakabeppu, Interferometric measurement of the liquid-phase temperature field around an isolated boiling bubble, J. Therm. Sci. Technol. 7 (3) (2012) 463-474.

[15] S. Moghaddam, K. Kiger, Physical mechanisms of heat transfer during single bubble nucleate boiling of FC-72 under saturation conditions-I. Experimental investigation, Int. J. Heat Mass Transfer 52 (2009) 1284-1294.

[16] N. Schweizer, P. Stephan, Experimental study of bubble behavior and local heat flux in poolboiling under variable gravitational conditions, Multiphase Sci. Technol. 21 (2009) 329-350.

[17] G. Son, V.K. Dhir, Dynamics and heat transfer associated with a single bubble during nucleate boiling on a horizontal surface, J. Heat Transfer 121 (1999) 623-631.

[18] G. Son, N. Ramanujapu, V.K. Dhir, Numerical simulation of bubble merger process on a single nucleation site during pool nucleate boiling, J. Heat Transfer 124 (2002) 51-62.

[19] D. Li, V.K. Dhir, Numerical study of a single bubble sliding on a downward facing heated surface, J. Heat Transfer 129 (7) (2007) 877-883.

[20] G. Son, Numerical study on a sliding bubble during pool nucleate boiling KSME Int. J. 15 (7) (2001) 931-940.

[21] J.F. Zhao, Z.D. Li, L. Zhang, Numerical simulation on single bubble pool boiling in different gravity conditions, Chin. J. Space Sci. 32 (4) (2012) 537-543.
[22] C. Kunkelmann, P. Stephan, Numerical simulation of the transient heat transfer during nucleate boiling of refrigerant HFE-7100, Int. J. Refrig. 33 (2010) 1221 1228.

[23] E. Aktinol, V.K. Dhir, Numerical simulation of nucleate boiling phenomenon coupled with thermal response of the solid, Microgravity Sci. Technol. 24 (2012) 255-265

[24] L. Zhang, Z.D. Li, K. Li, H.X. Li, J.F. Zhao, Influence of transient thermal response of solid wall on bubble dynamics in pool boiling, J. Comput. Multiphase Flows 6 (3) (2014) 313-327.

[25] L. Zhang, Z.D. Li, K. Li, H.X. Li, J.F. Zhao, Influence of heater thermal capability on bubble dynamics and heat transfer in nucleate pool boiling, Appl. Therm. Eng. (2014), http://dxdoi.org/10.1016/j.applthermaleng.2014.11.080 (in print).

[26] L. Zhang, Study on the thermal dynamical characteristics of fluid particles in microgravity (Ph.D. thesis), Institute of Mechanics, Chinese Academy of Sciences, Beijing, 2014.

[27] W.R. Hu, J.F. Zhao, M. Long, X.W. Zhang, Q.S. Liu, M.Y. Hou, Q. Kang, Y.R. Wang, S.H. Xu, W.J. Kong, H. Zhang, S.F. Wang, Y.Q. Sun, H.Y. Hang, Y.P. Huang, W.M. Cai, Y. Zhao, J.W. Dai, H.Q. Zheng, E.K. Duan, J.F. Wang, Space program SJ-10 of microgravity research, Microgravity Sci. Technol. 26 (2014) 159-169.

[28] D.Q. Nguyen, R.P. Fedkiw, M. Kang, A boundary condition capturing method for incompressible flame discontinuities, J. Comput. Phys. 172 (1) (2001) 71-98.

[29] B.B. Mikic, W.M. Rohsenhow, A new correlation of pool boiling data including the effect of heating surface characteristics, J. Heat Transfer 9 (1969) $245-250$.

[30] W.M. Kays, M.E. Grawford, Convective Heat and Mass Transfer, McGraw-Hill, New York, 1980.

[31] Y.Y. Hsu, On the size range of active nucleation cavities on a heating surface ASME J. Heat Transfer 84 (1962) 207-216.

[32] W. Mulder, S. Osher, Computing interface motion in compressible gas dynamics, J. Comput. Phys. 100 (2) (1992) 209-228.

[33] J.F. Zhao, Z.D. Li, H.X. Li, J. Li, Thermocapillary migration of deformable drops at moderate to large Marangoni number in microgravity, Microgravity Sci. Technol. 22 (3) (2010) 295-303.

[34] J.F. Zhao, L. Zhang, Z.D. Li, W.T. Qin, Topological structure evolvement of flow and temperature fields in deformable drop Marangoni migration in microgravity, Int. J. Heat Mass Transfer 54 (2011) 4655-4663.

[35] A. Sielaff, J. Dietl, S. Herbert, P. Stephan, The influence of system pressure on bubble coalescence in nucleate boiling, Heat Transfer Eng. 35 (5) (2014) 420 429.

[36] N. Zuber, Nucleate boiling: the region of isolated bubbles and the similarity with natural convection, Int. J. Heat Mass Transfer 6 (1) (1963) 53-79.

[37] H.A. Johnson, Transient boiling heat transfer to water, Int. J. Heat Mass Transfer 14 (1971) 67-82.

[38] J.F. Zhao, J. Li, N. Yan, S.F. Wang, Bubble behavior and heat transfer in quasisteady pool boiling in microgravity, Microgravity Sci. Technol. 21 (1) (2009) 175-183.

[39] A. Sakurai, M. Shiotsu, K. Hata, Y. Takeuchi, Quasi-steady nucleate boiling and its life caused by large stepwise heat input in saturated pool liquid He I, Cryogenics 29 (6) (1989) 597-601. 